\title{
Underwater acoustic tank evaluation of acoustic properties of samples using spectrally dense signals
}

\author{
C. Ranz Guerra and P. Cobo Parra \\ Instituto de Acústica. C.S.I.C., C/Serrano 144. 28006 Madrid. Spain.
}

Summary: This paper deals with exploring the availability and reliability of subtraction techniques, based on spectrally dense signals such as Maximun Length Sequences, in evaluating the echo reduction losses of samples of interest in underwater acoustics. These techniques, first employed in air acoustics, were adapted to the underwater environment. Experimental results show how a measurement can be accomplished and the limitations produced by the actual available combination of soft, hardware and tank and sample dimensions.

\section{INTRODUCTION}

The evaluation, within a limited underwater tank, of the relevant acoustical properties of materials for underwater use, can be a complex task. The constraints imposed by the sample dimensions and the tank dimensions have to be carefully considered in order to clearly separate the four main signal components of interest: the direct, the reflected/transmitted, the boundary reflected and the edge difracted signals; figure 1, shows those signals while measuring the reflective response of a sample, $2.0 \mathrm{~m}$ by $2.0 \mathrm{~m}$, of high density polyetilene, HML 500 . The time axis gives the relative distance between emitter, receiver, sample and

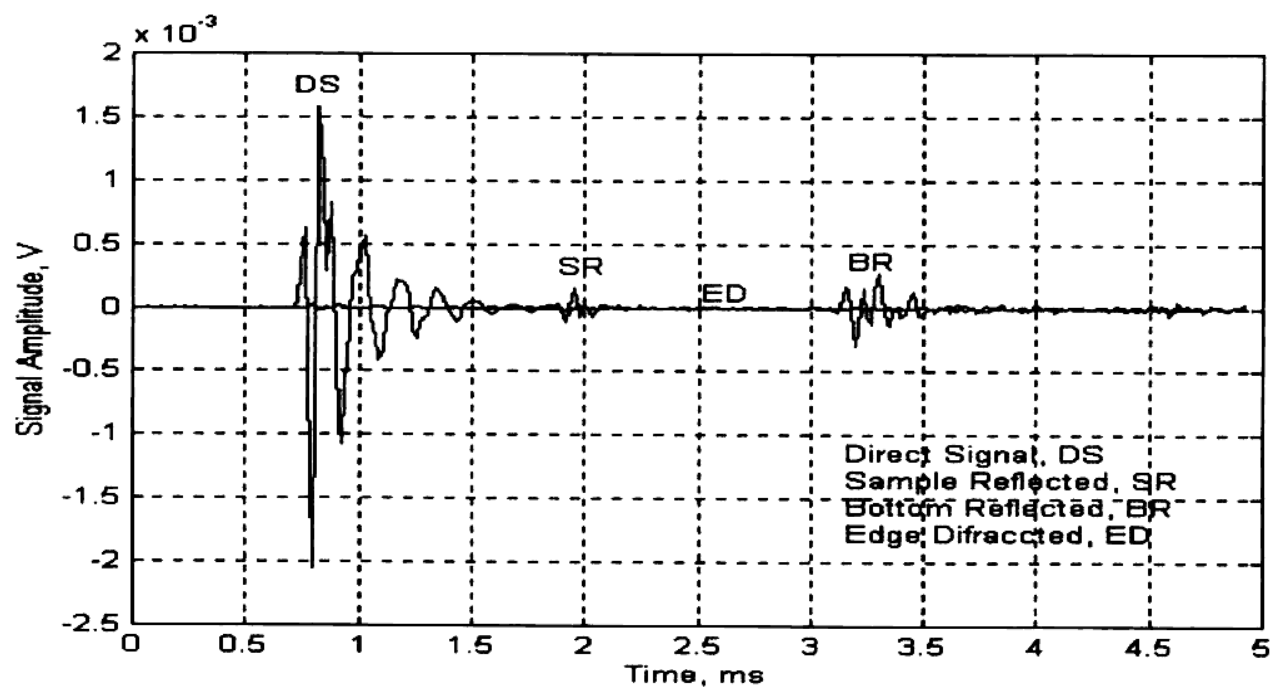

Fig. 1. Actual signal contributions in a reflection losses measurement

boundaries. The underwater tank at the Instituto de Acústica measures $4.5 \mathrm{~m} \mathrm{x} 4.5 \mathrm{~m} \mathrm{x} 7.5 \mathrm{~m}$. The position of the sensors is externally controlled by a computer. 
The aim of the work was to establish a simplified way of getting the reflective response of samples underwater. The reflection losses were given by,

$$
R L(f)=\frac{\left|F\left\{p_{s}(t) w_{s}(t)\right\}\right|^{2}}{\left|F\left\{p_{d}(t) w_{d}(t)\right\}\right|^{2}}
$$

being: $p_{s}(t)$, and $p_{d}(t)$, the pressures, picked up by the hydrophone, after being reflected at the sample and the pressure signal before reaching the sample; the respective smoothing window functions are $\mathrm{w}_{\mathrm{s}}(\mathrm{t})$, and $\mathrm{w}_{\mathrm{d}}(\mathrm{t})$. $F$ stands for Fourier Transform. The effect of windowing implies truncation, specially at low frequencies, so when starting from traces as the one given by figure 1 , great attention should be given to the windowing process, first by not letting out valid information, and by not letting in contaminated data from other specific origins. The window length (its inverse), on the other hand, sets the low frequency limit in the experience. The solution always is a compromise between all these factors. To improve the results is adequate to apply the subtraction method that implies two experimental settings. The first comes dictated by the solutions to the geometrical problem of locating the sample, emitter and receiver, within the tank, in such a way as to have all the signals identified and separated from each other. If the frequency response of the emitter is known, the frequency range of interest and hence the most adequate pulse length is also determined; figure 1, presents the most significant parts of the signal recorded. The second setting keeps the emitter and receiver in their previous locations and removes the sample; in this case the direct signal and the reflection at the boundaries are only present, figure $2-a$. Figure $2-b$ shows the sample reflected signal after the direct (figure 2-a) being removed from the whole result, figure 1.
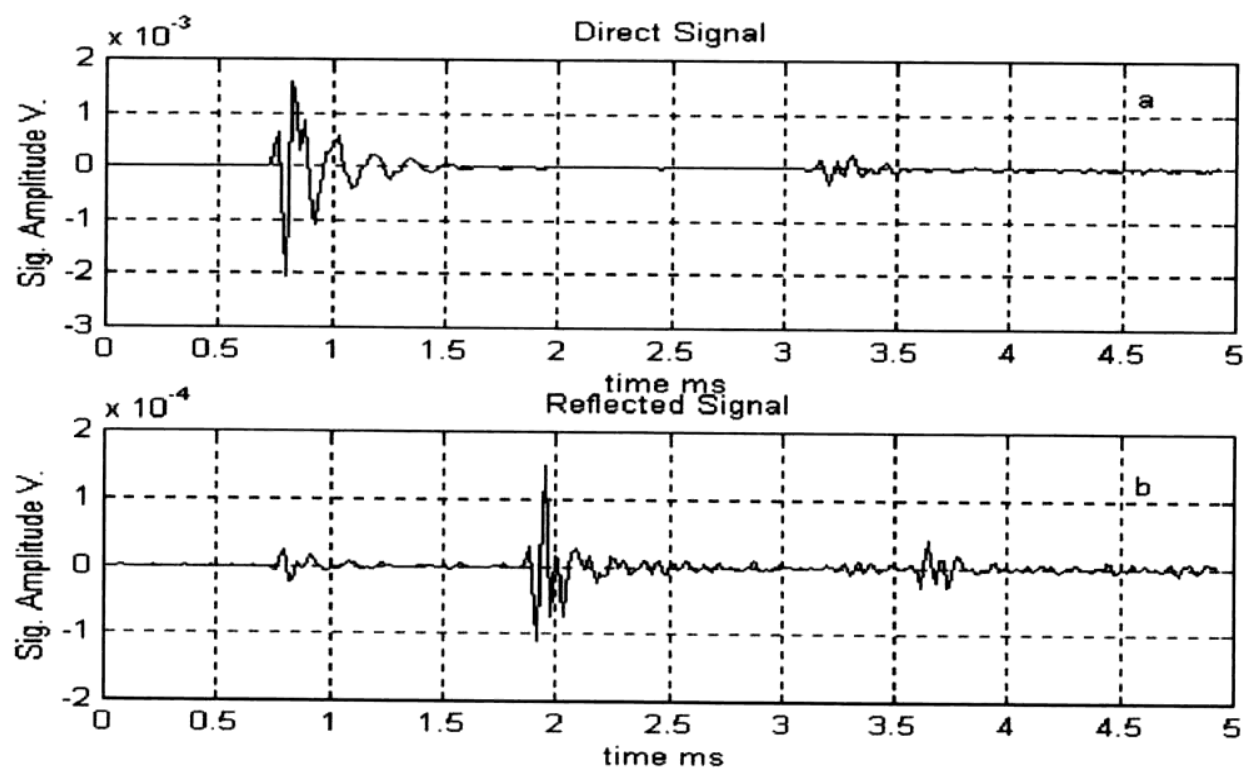

Figure2. Direct(a) \& Sample Reflected (b) signals 


\section{RESULTS}

The authors have shown the importance of using spectrally dense signals, MLS signals, in experiments underwater,C. Ranz and P. Cobo (1998). Maximun Length Sequences, are a kind of pseudorandom periodic signals whose suitability for being used underwater, comes from the extremely short time needed to get data characterizing acoustic transducers and acoustic media. The MLS signals are very immune to noise, they present very low distortion and a high time-bandwidth product, D. Rife and J Vanderkooy (1989). Spectrally dense signals have been used in air acoustics for several years since, P. Cobo et al. (1997), but is not until very recently that they become a basic part of the framework in underwater measurements. The experimental setup described in this paper makes use of these signals. The geometry of the measurement suggests the presence of the "edge difraccted", ED, signal at a distance of $0.5 \mathrm{~ms}$ after the reflected signal has reached the hydrophone. As a consequence the window (over the reflected signal) to get rid of the "edge difraccted" was to measure not more than 0.5 $\mathrm{ms}$; given the nature of the material under test its ED contribution is unimportant, figure 2-b, and practically the window length that really matters, is the traveling time between the Direct and the Sample Reflected signals. This length is $t_{w}=1.02$ ms. Previous experiences, about similar measures in air acoustics, have shown that a suitable window function is the "Adrienne" window, that is made from a combination of three Blackman-Harris windows, J.P.Clairbois et al. (1998). Figures 3-a and 3-b, show the power spectrum of both signals, once windowed, and finally figure 4-a and 4-b, presents the reflection losses, RL.
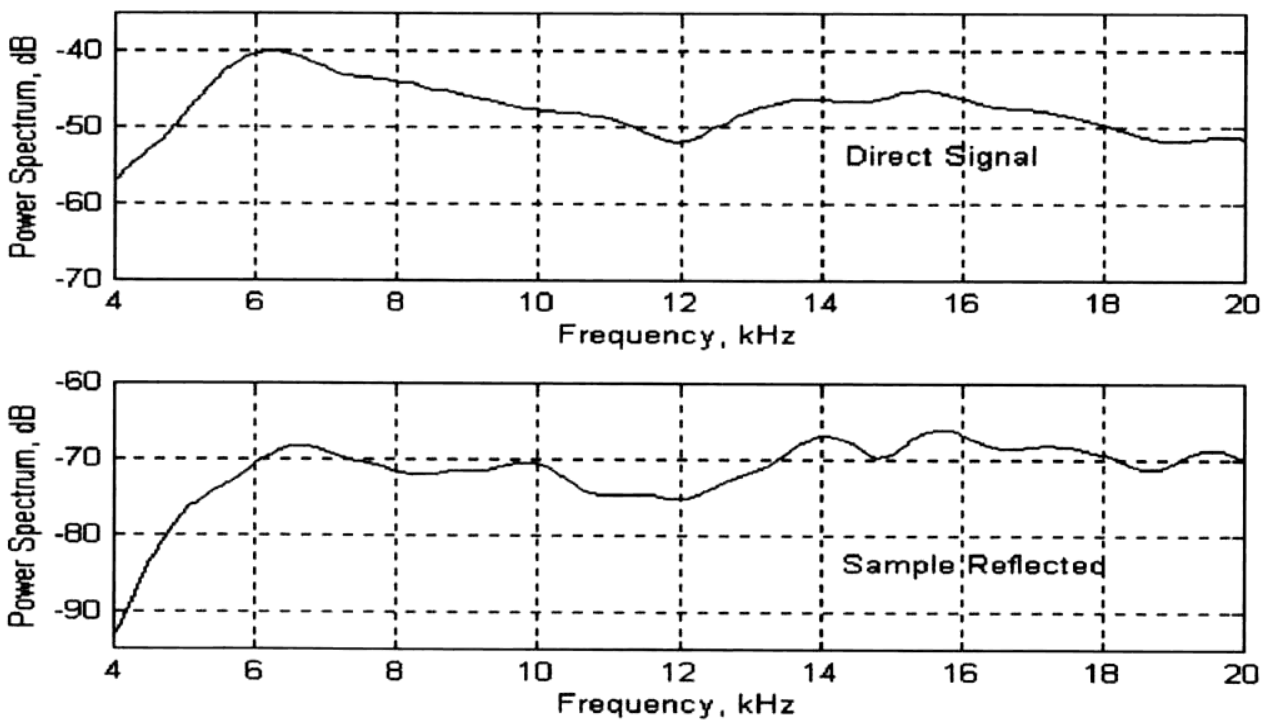

Figure 3. Power Spectrum of Direct (a) and Reflected at the sample (b).

\section{CONCLUSIONS}

The evaluation of the normal incidence reflective response of materials for use underwater, has been obtained making use of the spectrally dense signals. The method is very easy implemented and in consequence it was incorporated to the renewed software of the underwater acoustic tank laboratory. The result comes limited because the limited dimensions 

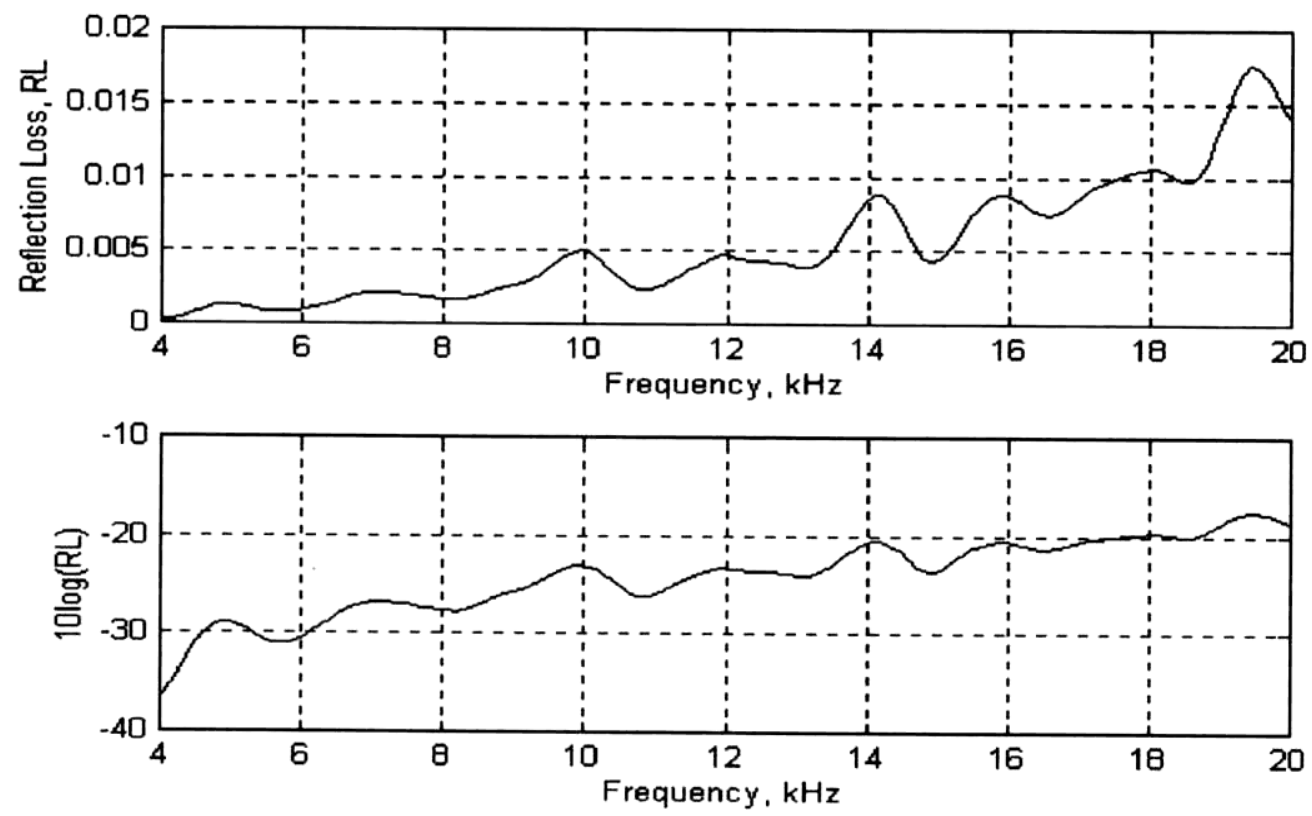

Figure 4. Reflective response of the sample

of the sample and those of the tank; the emitter frequency response also sets a maximun value to the pulse length to be used in the experiment and hence this property limits, in distance and frequency as well, the measurements.

\section{ACKNOWLEDGEMENT}

The authors wish to acknowledge the help given by the CICYT, Project AMB97-1175-C0301, for having the opportunity to use the software, hardware and related facilities in carrying out the work described by this paper.

\section{REFERENCES}

Clairbois, J.P., et al. " $A$ new in situ method for the acoustic performance of road traffic noise reducing devices". Proc. ICA 98. Seattle, USA. pp:471-472. 1998.

Cobo, P. et al., "Reflection loss measurements on flat and non flat samples using MLS method with signals subtraction techniques”. Tecniacústica 97, pp 173-176. Oviedo. Noviembre 1997.

Ranz, C. and Cobo, P. "Métodos de medida vía "secuencias de máxima longitud", (MLS), incorporados al laboratorio tanque de Hidroacústica del Instituto de Acústica”. ACÚSTICA 98. Lisboa. 1998. Pp: 249-252.

Rife, D. and Vanderkooy, J., "Transfer-function measurement with maximun length sequences”, J. Audio Eng, Soc, Vol 37, 6, Junio 1989. 